\title{
THE RECONSTRUCTION OF OBSTACLES IN A WAVEGUIDE USING FINITE ELEMENTS*
}

\author{
Ruming Zhang and Jiguang Sun \\ Department of Mathematical Sciences, Michigan Technological University, Michigan, USA \\ Email: rumingz@mtu.edu, jiguangs@mtu.edu
}

\begin{abstract}
This paper concerns the reconstruction of a penetrable obstacle embedded in a waveguide using the scattered data due to point sources, which is formulated as an optimization problem. We propose a fast reconstruction method based on a carefully designed finite element scheme for the direct scattering problem. The method has several merits: 1) the linear sampling method is used to quickly obtain a good initial guess; 2) finite Fourier series are used to approximate the boundary of the obstacle, which is decoupled from the boundary used by the finite element method; and 3) the mesh is fixed and hence the stiffness matrix, mass matrix, and right hand side are assembled once and only minor changes are made at each iteration. The effectiveness of the proposed method is demonstrated by numerical examples.
\end{abstract}

Mathematics subject classification: 78A46, 65M32, 65M60.

Key words: Inverse scattering problem, Waveguides, Finite element method.

\section{Introduction}

There are only a few works in literature devoted to the reconstruction of obstacles embedded in periodic structures. In [18], Xu et al. applied a method using generalized dual space indicator for an obstacle in a shallow water waveguide. Dediu and McLaughlin [8] proposed an eigensystem decomposition to recover weak inhomogeneities in a planar waveguide from far-field data. In $[4,5]$, Bourgeois and Lunéville employed the linear sampling method to reconstruct sound soft obstacles as well as cracks in a planar waveguide from near-field data. A factorization method is used for the inverse scattering problems in a 3D planar waveguide by Arens et al. [1]. For the reconstruction of Dirichlet and impedance obstacles, reverse time migration was employed [6] by Chen and Huang. Recently, a direct sampling method and a multilevel method were introduced in $[12,13]$ to reconstruct a penetrable inhomogeneous medium embedded in a 3D waveguide [14] and in the stratified ocean waveguide [15], respectively. Note that [4] contains a uniqueness result that the scattered waves correspond to infinitely many incident fields uniquely determine a sound-soft obstacle.

We note that, for a non-planar waveguide, the problem becomes much harder due to the lack of efficient numerical methods for the direct scattering problems. In [17], based on the recursive doubling procedure for periodic structures [9], Sun and Zheng employed the linear sampling method to reconstruct sound soft obstacles using near field data. A similar treatment, but using a different numerical technique for the scattering problem, can be found in [3].

In this paper, we propose an efficient finite element optimization method to reconstruct a penetrable obstacle embedded in a planar waveguide. Consider the waveguide given by

\footnotetext{
* Received February 28, 2016 / Revised version received September 18, 2016 / Accepted October 8, 2016 /

Published online October 11, 2017 /
} 
$\Omega=\mathbb{R} \times[0, H], H>0$. The lower and upper boundaries are $\Sigma_{-}=\mathbb{R} \times\{0\}$ and $\Sigma_{+}=\mathbb{R} \times\{H\}$, which are assumed to be sound-hard. Let $D$ be a star-shaped domain with Lipschitz continuous boundary $\partial D$. Then there exists a point $\left(z_{1}, z_{2}\right)$ and a periodic function $r$ such that the boundary $\partial D$ can be represented as

$$
\partial D:=\left\{\left(z_{1}, z_{2}\right)+r(t)(\cos t, \sin t) \mid 0 \leqslant t<2 \pi\right\} .
$$

The inverse problem is to determine the location and shape of $D$ from the measured scattered field due to incident waves by point sources. In particular, the point sources locate on $\Gamma_{i}$ and the scattered field is measured on $\Gamma_{m}$, where $\Gamma_{i}$ and $\Gamma_{m}$ are line segments (see Figure 1.1). For example, $\Gamma_{i}=\Gamma_{m}=\Gamma_{1} \cup \Gamma_{\text {? }}$.

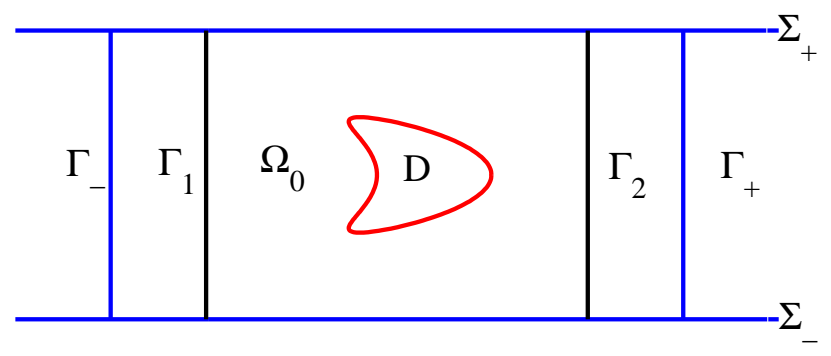

Fig. 1.1. The physical configuration of the scattering problem.

We assume that $r$ can be represented by a finite Fourier series. The inverse problem is formulated as an optimization problem, which is solved by a quasi-Newton method. The initial guess is obtained using the linear sampling method [17]. Since a lot of direct scattering problems need to be solved numerically, we design an efficient finite element method using a fixed mesh (see [19]). The stiffness matrix, mass matrix, and right-hand side only need to be assembled once at the beginning of the numerical procedure. Only minor changes are necessary in the subsequent iterations. Multiple frequency data are used to obtain a better reconstruction (see, e.g., $[2,16,19])$.

The rest of paper is organized as follows. Section 2 introduces the direct scattering problem. In Section 3, the inverse problem is formulated as an optimization problem, and the Fréchet derivative of the target function is studied. In Section 4, we present the reconstruction method in detail. Section 5 contains numerical results.

\section{The Direct Problem}

Let $k=w / c$ be the wavenumber, where $w$ is the frequency and $c$ is the speed of sound. For planar waveguides, there exist eigenvalues and eigenfunctions of Sturm-Liouville type (see [8]) given by

$$
k_{n}=\frac{n \pi}{H}, \quad \theta_{n}\left(x_{2}\right)= \begin{cases}\sqrt{\frac{1}{H}}, & n=0, \\ \sqrt{\frac{2}{H}} \cos \left(\frac{n \pi}{H} x_{2}\right), & n \geqslant 1 .\end{cases}
$$

The incident field $u^{i}:=G(\cdot, y)$, generated by a point source located on $\Gamma_{i}$, is defined as (see [4])

$$
G(x, y)=\sum_{n \in \mathbb{N}} \frac{e^{i \beta_{n}\left|x_{1}-y_{1}\right|}}{2 i \beta_{n}} \theta_{n}\left(x_{2}\right) \theta_{n}\left(y_{2}\right),
$$


where $\beta_{n}=\sqrt{k^{2}-k_{n}^{2}}, \operatorname{Re} \beta_{n} \geqslant 0, \operatorname{Im} \beta_{n} \geqslant 0$. The index of refraction $n$ satisfies

$$
n= \begin{cases}n_{1}, & \text { in } \Omega \backslash D, \\ n_{2}, & \text { in } D,\end{cases}
$$

where $n_{1}=1, n_{2} \neq n_{1}$, Re $n_{2}>0, \operatorname{Im} n_{2} \geqslant 0$.

We define two line segments (see Figure 1.1)

$$
\Gamma_{ \pm}=\left\{\left( \pm s, x_{2}\right), x_{2} \in(0, H), s>\max _{t \in[0,2 \pi)}\left\{\left|z_{1}+r(t) \cos t\right|\right\}\right\} .
$$

Hence $\Omega_{0}=(-s, s) \times(0, H)$ is a rectangle containing $D$. Let $T_{ \pm}$be the Dirichlet-to-Neumann maps on $\Gamma_{ \pm}$given by

$$
T_{ \pm} v=\sum_{n \in \mathbb{N}} i \beta_{n}\left(v, \theta_{n}\right)_{\Gamma_{ \pm}} \theta_{n}, \text { where }(\cdot, \cdot)_{\Gamma_{ \pm}} \text {is the inner product on } L^{2}\left(\Gamma_{ \pm}\right) .
$$

Then the operators $T_{ \pm}$satisfy the following lemma, whose proof is straightforward and thus omitted.

Lemma 2.1. Assume that $w \in H^{1 / 2}\left(\Gamma_{ \pm}\right)$. Then

$$
\left(T_{ \pm}\right)^{*} w=\overline{\left(T_{ \pm}\right) \bar{w}}
$$

Let $u$ be the total field and $u^{i}$ be the incident field. The direct problem is to find the scattered field $u^{s}$ such that

$$
\begin{array}{ll}
\triangle u^{s}+k^{2} n u^{s}=k^{2}(1-n) u^{i}, & \text { in } \Omega_{0}, \\
\frac{\partial u^{s}}{\partial x_{2}}=0, & \text { on } \Sigma_{ \pm}, \\
\frac{\partial u^{s}}{\partial x_{1}}=-T_{-} u^{s}, & \text { on } \Gamma_{-}, \\
\frac{\partial u^{s}}{\partial x_{1}}=T_{+} u^{s}, & \text { on } \Gamma_{+} .
\end{array}
$$

Note that $u^{s}=u-u^{i}$, where $u$ is the total field.

The variational formulation of $(2.2)$ is to find $u^{s} \in H^{1}\left(\Omega_{0}\right)$ such that

$$
-\int_{\Omega_{0}} \nabla u^{s} \cdot \overline{\nabla \phi}+k^{2} \int_{\Omega_{0}} n u^{s} \bar{\phi}+\int_{\Gamma_{-}} T_{-} u^{s} \bar{\phi} d s+\int_{\Gamma_{+}} T_{+} u^{s} \bar{\phi} d s=k^{2} \int_{D}(1-n) u^{i \bar{\phi}}
$$

for $\phi \in H^{1}\left(\Omega_{0}\right)$. For simplicity, we omit $d x$ in the volume integral throughout the paper. The well-posedness of the direct scattering problem is shown in the following theorem. Its proof is standard using the analytic Fredholm theory and thus omitted.

Theorem 2.1. There exists a countable subset $\mathbb{K} \subset \mathbb{R}^{+}$with at most one accumulation $+\infty$ such that, for a fixed $k \in \mathbb{R}^{+} \backslash \mathbb{K}$, the variational problem (2.3) is uniquely solvable in $H^{1}\left(\Omega_{0}\right)$ and the scattered field $u^{s}$ satisfies

$$
\left\|u^{s}\right\|_{H^{1}\left(\Omega_{0}\right)} \leqslant C\left\|u^{i}\right\|_{L^{2}\left(\Omega_{0}\right)},
$$

where $C$ is some constant independent of $u^{i}$. 
Remark 2.1. In the rest of the paper, we assume that the wavenumber $k \notin \mathbb{K}$.

Let $N$ be a fixed positive integer and $\mathbf{C}=\left(c_{0}, c_{1}, \ldots, c_{2 N}\right) \in \mathbb{R}^{2 N+1}$. Let the function $r(t)$ be defined as

$$
r(t)=\sum_{l=0}^{2 N} c_{l} \phi_{l}(t), \quad t \in[0,2 \pi)
$$

where $\phi_{l}$ 's are the trigonometric basis functions

$$
\phi_{0}=1, \quad \phi_{l}=\cos (l t), \quad \phi_{l+N}=\sin (l t), \quad l=1, \ldots, N .
$$

We define an operator

$$
f: \quad \mathbf{C} \longrightarrow r(t)
$$

and the space

$$
X_{N}:=\left\{f(\mathbf{C}), \mathbf{C} \in \mathbb{R}^{2 N+1}\right\} .
$$

We assume that, for a given domain $D$, there exists $\left(z_{1}, z_{2}\right) \in \Omega_{0}$ such that

$$
\partial D \in\left\{\left(z_{1}, z_{2}\right)+r(t)(\cos t, \sin t), r \in X_{N}\right\} .
$$

Define the scattering operator $S$ such that

$$
\begin{aligned}
S: \quad X_{N} & \longrightarrow H^{1}\left(\Omega_{0}\right), \\
r & \left.\mapsto u^{s}\right|_{\Gamma_{m}} .
\end{aligned}
$$

To show that $S$ is Fréchet differentiable (see $[10,11]$ ), we need the following lemma, whose proof is obvious.

Lemma 2.2. Assume that $\mathbf{h}(t)=h(t)(\cos t, \sin t), h(t) \in X_{N}$. Let $\eta=\|h(t)\|_{L_{\infty}^{\infty}[0,2 \pi]}$. Furthermore, for a fixed $r(t)$, let $D$ be the domain with $\partial D=\left(z_{1}, z_{2}\right)+r(t)(\cos t, \sin t)$ and $D^{h}$ be the domain with $\partial D^{h}=\left(z_{1}, z_{2}\right)+r(t)(\cos t, \sin t)+\mathbf{h}$. Let $D_{c}=\left(D \backslash D^{h}\right) \cup\left(D^{h} \backslash D\right)$. Then $\left|D_{c}\right| \leq C \eta$, where $\left|D_{c}\right|$ is the area of $D_{c}$, for some constant $C$ independent of $h(t)$.

Let $\nu$ be the unit outward normal to $\partial D$. The following lemma gives the Fréchet derivative of $S$.

Lemma 2.3. The scattering operator $S$ is Fréchet differentiable, and its Fréchet derivative, denoted by $F S$, is given by

$$
F S(r) h=\left.v\right|_{\Gamma_{m}}, \quad \text { where } r, h \in X_{N} .
$$

Here $v$ satisfies (2.2) and the following boundary conditions on $\partial D$

$$
\left.v\right|_{-}-\left.v\right|_{+}=0,\left.\quad \frac{\partial v}{\partial \nu}\right|_{-}-\left.\frac{\partial v}{\partial \nu}\right|_{+}=k^{2}\left(n_{2}-1\right)(\mathbf{h} \cdot \nu) u,
$$

where $u$ is the total field. 
Proof. Let $u^{h}$ be the total field for $D^{h}$ and $u^{s, h}$ be the corresponding scattered field. Denote by $w=u^{h}-u=u^{s, h}-u^{s}$. For any $\phi \in H^{1}\left(\Omega_{0}\right), w$ satisfies the following variational form

$$
\begin{aligned}
& -\int_{\Omega_{0}} \nabla w \cdot \overline{\nabla \phi}+k^{2} \int_{\Omega_{0}}\left(n^{h} u^{s, h}-n u^{s}\right) \bar{\phi}+\int_{\Gamma_{-}} T_{-} w \bar{\phi} d s+\int_{\Gamma_{+}} T_{+} w \bar{\phi} d s \\
= & k^{2} \int_{\Omega_{0}}\left(n-n^{h}\right) u^{i} \bar{\phi} .
\end{aligned}
$$

From the facts that $u=u^{s}+u^{i}$ and $u^{h}=u^{s, h}+u^{i}$, the following equation holds

$$
-\int_{\Omega_{0}} \nabla w \cdot \overline{\nabla \phi}+k^{2} \int_{\Omega_{0}} n w \bar{\phi}+\int_{\Gamma_{-}} T_{-} w \bar{\phi} d s+\int_{\Gamma_{+}} T_{+} w \bar{\phi} d s=k^{2} \int_{\Omega_{0}}\left(n-n^{h}\right) u^{h} \bar{\phi} .
$$

Note that $\left|n^{h}-n\right|=\left|n_{2}-1\right| \neq 0$ only in $D_{c}$. From Lemma 2.2, we have that

$$
\left|k^{2} \int_{\Omega_{0}}\left(n-n^{h}\right) u^{h} \bar{\phi}\right| \leqslant C\left|D_{c}\right|\left\|u^{h}\right\|_{H^{1}\left(\Omega_{0}\right)}\|\phi\|_{H^{1}\left(\Omega_{0}\right)} \leqslant C \eta\left\|u^{h}\right\|_{H^{1}\left(\Omega_{0}\right)}\|\phi\|_{H^{1}\left(\Omega_{0}\right)} .
$$

From Theorem 2.1,

$$
\|w\|_{H^{1}\left(\Omega_{0}\right)} \leqslant C \eta\left\|u^{h}\right\|_{H^{1}\left(\Omega_{0}\right)} \leqslant C \eta\left\|u^{i}\right\|_{L^{2}\left(\Omega_{0}\right)} .
$$

It is clear that $v$ satisfies the following equations

$$
\begin{aligned}
& -\int_{D} \nabla v \cdot \overline{\nabla \phi}+k^{2} \int_{D} n v \bar{\phi}+\left.\int_{\partial D} \frac{\partial v}{\partial \nu}\right|_{-} \bar{\phi} d s=0, \\
& -\int_{\Omega_{0} \backslash D} \nabla v \cdot \overline{\nabla \phi}+k^{2} \int_{\Omega_{0} \backslash D} n v \bar{\phi}-\left.\int_{\partial D} \frac{\partial v}{\partial \nu}\right|_{+} \bar{\phi}+\int_{\Gamma_{-}} T_{-} v \bar{\phi}+\int_{\Gamma_{+}} T_{+} v \bar{\phi} d s=0,
\end{aligned}
$$

in $D$ and $\Omega_{0} \backslash D$, respectively. Using the boundary condition (2.5), $v$ satisfies

$$
\begin{aligned}
& -\int_{\Omega_{0}} \nabla v \cdot \overline{\nabla \phi}+k^{2} \int_{\Omega_{0}} n v \bar{\phi}+\int_{\Gamma_{-}} T_{-} v \bar{\phi} d s+\int_{\Gamma_{+}} T_{+} v \bar{\phi} d s \\
= & k^{2} \int_{\partial D}\left(1-n_{2}\right)(\mathbf{h} \cdot \nu) u \bar{\phi} d s .
\end{aligned}
$$

Then $w-v$ satisfies

$$
\begin{aligned}
& -\int_{\Omega_{0}} \nabla(w-v) \cdot \overline{\nabla \phi}+k^{2} \int_{\Omega_{0}} n(w-v) v \bar{\phi}+\int_{\Gamma_{-}} T_{-}(w-v) \bar{\phi} d s+\int_{\Gamma_{+}} T_{+}(w-v) \bar{\phi} d s \\
= & k^{2} \int_{\Omega_{0}}\left(n-n^{h}\right) u^{h} \bar{\phi}-k^{2} \int_{\partial D}\left(1-n_{2}\right)(\mathbf{h} \cdot \nu) u \bar{\phi} d s .
\end{aligned}
$$

The right hand side satisfies

$$
\begin{aligned}
& \left|k^{2} \int_{\Omega_{0}}\left(n-n^{h}\right) u^{h} \bar{\phi}-k^{2} \int_{\partial D}\left(1-n_{2}\right)(\mathbf{h} \cdot \nu) u \bar{\phi} d s\right| \\
\leqslant & C\left|\int_{\Omega_{0}}\left(n-n^{h}\right) w \bar{\phi}\right|+C\left|\int_{\Omega_{0}}\left(n-n^{h}\right) u \bar{\phi}-\int_{\partial D}\left(1-n_{2}\right)(\mathbf{h} \cdot \nu) u \bar{\phi} d s\right| .
\end{aligned}
$$

Let

$$
I=\left|\int_{\Omega_{0}}\left(n-n^{h}\right) w \bar{\phi}\right|, \quad I I=\left|\int_{\Omega_{0}}\left(n-n^{h}\right) u \bar{\phi}-\int_{\partial D}\left(1-n_{2}\right)(\mathbf{h} \cdot \nu) u \bar{\phi} d s\right| .
$$


From Lemma 2.2, I satisfies

$$
I \leqslant C \eta\|w\|_{H^{1}\left(D_{c}\right)}\|\phi\|_{H^{1}\left(D_{c}\right)} \leqslant C \eta^{2}\left\|u^{i}\right\|_{L^{2}\left(\Omega_{0}\right)}\|\phi\|_{H^{1}\left(D_{c}\right)} .
$$

For the second term, we have that

$$
\begin{aligned}
I I & =\left|\int_{D \backslash D^{h}}\left(n_{2}-1\right) u \bar{\phi}+\int_{D^{h} \backslash D}\left(1-n_{2}\right) u \bar{\phi}-\int_{\partial D}\left(1-n_{2}\right)(\mathbf{h} \cdot \nu) u \bar{\phi} d s\right| \\
& =\left|n_{2}-1\right|\left|\int_{D \backslash D^{h}} u \bar{\phi}-\int_{D^{h} \backslash D} u \bar{\phi}+\int_{\partial D}(\mathbf{h} \cdot \nu) u \bar{\phi} d s\right| .
\end{aligned}
$$

We define

$$
N(t)=\sqrt{\left[r^{\prime}(t) \cos t-r(t) \sin t\right]^{2}+\left[r^{\prime}(t) \sin t+r(t) \cos (t)\right]^{2}}
$$

Then

$$
\begin{aligned}
& d s=N(t) d t, \quad \nu=\frac{r^{\prime}(t)(\sin t,-\cos t)+r(t)(\cos t, \sin t)}{N(t)}, \\
& (\mathbf{h} \cdot \nu) d s=h(t)(\cos t, \sin t) \cdot\left[r^{\prime}(t)(\sin t,-\cos t)+r(t)(\cos t, \sin t)\right] d t=h(t) r(t) d t .
\end{aligned}
$$

Thus we obtain

$$
\int_{\partial D}(\mathbf{h} \cdot \nu) u \bar{\phi} d s=\int_{0}^{2 \pi}(u \cdot \bar{\phi})(r(t), t) h(t) r(t) d t .
$$

Since $u, \phi \in H^{1}\left(\Omega_{0}\right)$, there exist functions $\theta_{1}(\rho, t)$ and $\theta_{2}(\rho, t)$ such that

$$
\begin{aligned}
& u(\rho, t)=u(r(t), t)+(\rho-r(t)) \frac{\partial}{\partial \rho} u\left(\theta_{1}(\rho, t), t\right), \\
& \phi(\rho, t)=\phi(r(t), t)+(\rho-r(t)) \frac{\partial}{\partial \rho} \phi\left(\theta_{2}(\rho, t), t\right),
\end{aligned}
$$

where $\frac{\partial}{\partial \rho} u\left(\theta_{1}(\rho, t), t\right)$ and $\frac{\partial}{\partial \rho} \phi\left(\theta_{2}(\rho, t), t\right)$ are in $L^{2}\left(\Omega_{0}\right)$.

Substituting the above results in $I I$, we get that

$$
\begin{aligned}
& I I=-\int_{0}^{2 \pi} \int_{r(t)}^{r(t)+h(t)}(u \cdot \bar{\phi})(\rho, t) \rho d \rho d t+\int_{0}^{2 \pi}(u \cdot \bar{\phi})(r(t), t) h(t) r(t) d t \mid \\
&=\mid-\int_{0}^{2 \pi} \int_{r(t)}^{r(t)+h(t)}\left[u(r(t), t)+(\rho-r(t)) \frac{\partial}{\partial \rho} u\left(\theta_{1}(\rho, t), t\right)\right] \\
& \cdot\left[\bar{\phi}(r(t), t)+(\rho-r(t)) \frac{\partial}{\partial \rho} \bar{\phi}\left(\theta_{2}(\rho, t), t\right)\right] \rho d \rho d t+\int_{0}^{2 \pi}(u \cdot \bar{\phi})(r(t), t) h(t) r(t) d t \mid \\
& \leqslant\left|-\int_{0}^{2 \pi} \int_{r(t)}^{r(t)+h(t)}(u \cdot \bar{\phi})(r(t), t) \rho d \rho d t+\int_{0}^{2 \pi}(u \cdot \bar{\phi})(r(t), t) h(t) r(t) d t\right| \\
&+\left|\int_{D_{c}}(\rho-r(t)) u(r(t), t) \frac{\partial}{\partial \rho} \bar{\phi}\left(\theta_{2}(\rho, t), t\right)\right|+\left|\int_{D_{c}}(\rho-r(t)) \bar{\phi}(r(t), t) \frac{\partial}{\partial \rho} u\left(\theta_{1}(\rho, t), t\right)\right| \\
&+\left|\int_{D_{c}}(\rho-r(t))^{2} \frac{\partial}{\partial \rho} u\left(\theta_{1}(\rho, t), t\right) \frac{\partial}{\partial \rho} \bar{\phi}\left(\theta_{2}(\rho, t), t\right)\right| .
\end{aligned}
$$


Using Lemma 2.2 and the fact that $u, \bar{\phi} \in H^{1}\left(\Omega_{0}\right)$, we obtain that

$$
\begin{aligned}
I I \leqslant\left|\int_{0}^{2 \pi} \frac{1}{2}(u \cdot \bar{\phi})(r(t), t) h^{2}(t) d t\right|+\|u\|_{L^{\infty}\left(D_{c}\right)} \int_{D_{c}}|\rho-r(t)|\left|\frac{\partial}{\partial \rho} \phi\left(\theta_{2}(\rho, t), t\right)\right| \\
+\|\phi\|_{L^{\infty}\left(D_{c}\right)} \int_{D_{c}}|\rho-r(t)| \frac{\partial}{\partial \rho} u\left(\theta_{1}(\rho, t), t\right) \mid \\
\quad+\|\rho-r(t)\|_{L^{\infty}\left(D_{c}\right)}^{2} \int_{D_{c}}\left|\frac{\partial}{\partial \rho} u\left(\theta_{1}(\rho, t), t\right)\right|\left|\frac{\partial}{\partial \rho} \phi\left(\theta_{2}(\rho, t), t\right)\right| \\
\leqslant \pi \eta^{2}\|u \bar{\phi}\|_{L^{\infty}\left(\Omega_{0}\right)}+\|u\|_{L^{\infty}\left(D_{c}\right)}\|\rho-r(t)\|_{L^{2}\left(D_{c}\right)}\left\|\frac{\partial}{\partial \rho} \phi\left(\theta_{2}(\rho, t), t\right)\right\|_{L^{2}\left(D_{c}\right)} \\
\quad+\|\phi\|_{L^{\infty}\left(D_{c}\right)}\|\rho-r(t)\|_{L^{2}\left(D_{c}\right)}\left\|\frac{\partial}{\partial \rho} u\left(\theta_{1}(\rho, t), t\right)\right\|_{L^{2}\left(D_{c}\right)} \\
\quad+\eta^{2}\left\|\frac{\partial}{\partial \rho} \phi\left(\theta_{2}(\rho, t), t\right)\right\|_{L^{2}\left(D_{c}\right)}\left\|\frac{\partial}{\partial \rho} u\left(\theta_{1}(\rho, t), t\right)\right\|_{L^{2}\left(D_{c}\right)} \\
\leqslant C\left(\eta^{2}+\eta^{3 / 2}+\eta^{3 / 2}+\eta^{2}\right)\|u\|_{H^{1}\left(\Omega_{0}\right)}\|\phi\|_{H^{1}\left(\Omega_{0}\right)} \\
\leqslant C \eta^{3 / 2}\left\|u^{i}\right\|_{L^{2}\left(\Omega_{0}\right)}\|\phi\|_{H^{1}\left(\Omega_{0}\right)} .
\end{aligned}
$$

The right hand side of (2.6) satisfies

$$
\left|k^{2} \int_{\Omega_{0}}\left(n-n^{h}\right) u^{h} \bar{\phi}-k^{2} \int_{\partial D}\left(1-n_{2}\right)(\mathbf{h} \cdot \nu) u \bar{\phi}\right|=C \eta^{3 / 2}\|\phi\|_{H^{1}\left(\Omega_{0}\right)} .
$$

From Theorem 2.1, we have that

$$
\|w-v\|_{H^{1}\left(\Omega_{0}\right)}=O\left(\eta^{3 / 2}\right)=o(\eta)
$$

Hence we obtain

$$
\|w-v\|_{H^{1 / 2}\left(\Gamma_{m}\right)} \leqslant C\|w-v\|_{H^{1}\left(\Omega_{0}\right)}=o(\eta) .
$$

Consequently, $F S(g) h=\left.v\right|_{\Gamma_{m}}$ and the proof is complete.

Define the operator $\mathbf{N}$ on $\mathbb{R}^{2 N+1}$ such that

$$
\begin{aligned}
& \mathbf{N}: \quad \mathbb{R}^{2 N+1} \longrightarrow H^{1 / 2}\left(\Gamma_{m}\right) \\
& \mathbf{C}=\left(c_{0}, c_{1}, \ldots, c_{2 N}\right) \mapsto S\left[f\left(c_{0}, c_{1}, \ldots, c_{2 N}\right)\right] .
\end{aligned}
$$

It is obvious that the partial derivatives of $\mathbf{N}$ satisfy

$$
\frac{\partial}{\partial c_{l}} \mathbf{N}(\mathbf{C})=F S[f(\mathbf{C})] \frac{\partial}{\partial c_{l}} f(\mathbf{C})=F S[f(\mathbf{C})]\left[\phi_{l}(\cos t, \sin t)\right] .
$$

\section{The Inverse Problem}

Let the point sources locate on $\Gamma_{i}$. Given the measured scattered field $u^{\text {meas }}$ on $\Gamma_{m}$, the inverse problem we are interested in is to reconstruct the penetrable obstacle $D$ assuming $n_{2}$ is a known constant. Recall that $\partial D=\left\{\left(z_{1}, z_{2}\right)+r(t)(\cos t, \sin t)\right\}$ and $r(t)$ is approximated by the finite series $r(t)=\sum_{t=0}^{2 N} c_{l} \phi_{l}(t)$. The inverse problem can be described as follows. 
Inverse Problem (IP): Find $\mathbf{C}^{\mathbf{0}}=\left(c_{0}^{0}, \cdots, c_{2 N}^{0}\right) \in \mathbb{R}^{2 N+1}$ such that

$$
\mathbf{N}\left(\mathbf{C}^{\mathbf{0}}\right)=u^{\text {meas }} \quad \text { on } \Gamma_{m} .
$$

Define

$$
F(\mathbf{C} ; \gamma)=\left\|\mathbf{N}(\mathbf{C})-u^{\text {meas }}\right\|_{L^{2}\left(\Gamma_{m}\right)}^{2}+\gamma\|\mathbf{C}\|_{l^{2}}^{2},
$$

where $\gamma>0$ is the regularization parameter. The inverse problem can be formulated as follows.

Optimization Problem (OP): Find $\mathbf{C}^{0} \in \mathbb{R}^{2 N+1}$, such that

$$
\mathbf{C}^{\mathbf{0}}=\min _{\mathbf{C} \in \mathbb{R}^{2 N+1}} F(\mathbf{C} ; \gamma) .
$$

To solve $\mathbf{O P}$, we need the gradient of $F(\ldots ; \gamma)$, which is given in the following lemma.

Lemma 3.1. Let $\phi_{l}, l=0, \ldots, 2 N$, be the trigonometric basis functions defined in (2.4). It holds that

$$
\frac{\partial}{\partial c_{l}}\left(\left\|\mathbf{N}(\mathbf{C})-u^{\text {meas }}\right\|_{L^{2}\left(\Gamma_{m}\right)}^{2}\right)=2 \operatorname{Re}\left(M\left[\mathbf{N}(\mathbf{C})-u^{\text {meas }}\right] \cdot(\cos t, \sin t), \phi_{l}\right),
$$

where $M=(F S[f(\mathbf{C})])^{*}$ is the adjoint operator of $F S[f(\mathbf{C})]$.

Proof. Straightforward calculation gives that

$$
\begin{aligned}
& \frac{\partial}{\partial c_{l}}\left(\left\|\mathbf{N}(\mathbf{C})-u^{\text {meas }}\right\|_{L^{2}\left(\Gamma_{m}\right)}^{2}\right) \\
= & \frac{\partial}{\partial c_{l}}\left(\mathbf{N}(\mathbf{C})-u^{\text {meas }}, \mathbf{N}(\mathbf{C})-u^{\text {meas }}\right)_{L^{2}\left(\Gamma_{m}\right)} \\
= & 2 \operatorname{Re}\left(\mathbf{N}(\mathbf{C})-u^{\text {meas }}, \frac{\partial}{\partial c_{l}} \mathbf{N}(\mathbf{C})\right)_{L^{2}\left(\Gamma_{m}\right)} \\
= & 2 \operatorname{Re}\left(\mathbf{N}(\mathbf{C})-u^{\text {meas }}, F S[f(\mathbf{C})]\left[\phi_{l}(\cos t, \sin t)\right]\right)_{L^{2}\left(\Gamma_{m}\right)} \\
= & 2 \operatorname{Re}\left(M\left[\mathbf{N}(\mathbf{C})-u^{\text {meas }}\right], \phi_{l}(\cos t, \sin t)\right)_{L^{2}\left(X_{N}\right)} \\
= & 2 \operatorname{Re}\left(M\left[\mathbf{N}(\mathbf{C})-u^{\text {meas }}\right] \cdot(\cos t, \sin t), \phi_{l}\right)_{L^{2}[0,2 \pi]}
\end{aligned}
$$

This completes the proof of the lemma.

Recall that $\Gamma_{1}=\left\{-s_{0}\right\} \times(0, H)$ and $\Gamma_{2}=\left\{s_{0}\right\} \times(0, H)$. If both $\Gamma_{i}$ and $\Gamma_{m}$ are chosen to be $\Gamma_{1}=\left\{-s_{0}\right\} \times(0, H)$, one has the following lemma for the operator $M$.

Lemma 3.2. Let $\Omega_{0}=\left(-s_{0}, s_{0}\right) \times(0, H)$. For all $\phi \in H^{-1 / 2}\left(\Gamma_{1}\right)$, the operator $M$ is given by

$$
M(\phi)=-\left.\overline{k^{2}\left(n_{2}-1\right) u w}\right|_{\partial D}[(\cos t, \sin t) \cdot \nu],
$$

where $w$ satisfies the boundary value problem

$$
\begin{array}{ll}
\triangle w+k^{2} n w=0, & \text { in } \Omega_{0}, \\
\frac{\partial w}{\partial x_{2}}=0, & \text { on } \Sigma_{ \pm}, \\
T_{+} w-\frac{\partial w}{\partial x_{1}}=0, & \text { on } \Gamma_{2}, \\
T_{-} w+\frac{\partial w}{\partial x_{1}}=\bar{\phi}, & \text { on } \Gamma_{1} .
\end{array}
$$


Proof. For all $\phi \in H^{-1 / 2}\left(\Gamma_{1}\right)$, the boundary value problem (3.4) has a unique solution $w \in H^{1}\left(\Omega_{0}\right)$. Then for any $h \in X_{N}$,

$$
\begin{aligned}
\left(M^{*} \phi, h\right)_{X_{N}} & =(\phi, F S[f(\mathbf{C})](h))_{\Gamma_{1}}=(\phi, v)_{\Gamma_{1}}+(0, v)_{\Gamma_{2}} \\
& =\left(\overline{T_{-} w}+\frac{\partial \bar{w}}{\partial x_{1}}, v\right)_{\Gamma_{1}}+\left(\overline{T_{+} w}-\frac{\partial \bar{w}}{\partial x_{1}}, v\right)_{\Gamma_{2}} \\
& =\left(T_{-}^{*} \bar{w}+\frac{\partial \bar{w}}{\partial x_{1}}, v\right)_{\Gamma_{1}}+\left(T_{+}^{*} \bar{w}-\frac{\partial \bar{w}}{\partial x_{1}}, v\right)_{\Gamma_{2}} \\
& =\left(\bar{w}, T_{-} v\right)_{\Gamma_{1}}+\left(\frac{\partial \bar{w}}{\partial x_{1}}, v\right)_{\Gamma_{1}}+\left(\bar{w}, T_{+} v\right)_{\Gamma_{2}}-\left(\frac{\partial \bar{w}}{\partial x_{1}}, v\right)_{\Gamma_{2}} \\
& =\int_{\Gamma_{1}} \bar{w} \overline{T_{-} v} d s+\int_{\Gamma_{1}} \frac{\partial \bar{w}}{\partial x_{1}} \bar{v} d s+\int_{\Gamma_{2}} \bar{w} \overline{T_{+} v} d s-\int_{\Gamma_{2}} \frac{\partial \bar{w}}{\partial x_{1}} \bar{v} d s \\
& =-\int_{\Gamma_{1}} \bar{w} \frac{\partial \bar{v}}{\partial x_{1}} d s+\int_{\Gamma_{1}} \frac{\partial \bar{w}}{\partial x_{1}} \bar{v} d s+\int_{\Gamma_{2}} \bar{w} \frac{\partial \bar{v}}{\partial x_{1}} d s-\int_{\Gamma_{2}} \frac{\partial \bar{w}}{\partial x_{1}} \bar{v} d s .
\end{aligned}
$$

Applying the Green's formula in $D$ and $\Omega_{0} \backslash D$, we get

$$
0=\int_{D}(\bar{w} \triangle \bar{v}-\bar{v} \triangle \bar{w})=\int_{\partial D}\left(\left.\frac{\partial \bar{v}}{\partial \nu}\right|_{-} \bar{w}-\left.\bar{v}\right|_{-} \frac{\partial \bar{w}}{\partial \nu}\right) d s
$$

and

$$
\begin{aligned}
0 & =\int_{\Omega_{0} \backslash D}(\bar{w} \triangle \bar{v}-\bar{v} \triangle \bar{w}) \\
& =\int_{\Gamma_{2}}\left(\frac{\partial \bar{v}}{\partial x_{1}} \bar{w}-\bar{v} \frac{\partial \bar{w}}{\partial x_{1}}\right) d s-\int_{\Gamma_{1}}\left(\frac{\partial \bar{v}}{\partial x_{1}} \bar{w}-\bar{v} \frac{\partial \bar{w}}{\partial x_{1}}\right) d s-\int_{\partial D}\left(\left.\frac{\partial \bar{v}}{\partial \nu}\right|_{+} \bar{w}-\left.\bar{v}\right|_{+} \frac{\partial \bar{w}}{\partial \nu}\right) d s
\end{aligned}
$$

Using the above two equations and boundary conditions (2.5), we have

$$
\begin{aligned}
(\phi, F S[f(\mathbf{C})](h))_{\Gamma_{1}} & =\int_{\partial D}\left(\left.\frac{\partial \bar{v}}{\partial \nu}\right|_{+} \bar{w}-\left.\bar{v}\right|_{+} \frac{\partial \bar{w}}{\partial \nu}\right) d s-\int_{\partial D}\left(\left.\frac{\partial \bar{v}}{\partial \nu}\right|_{-} \bar{w}-\left.\bar{v}\right|_{-} \frac{\partial \bar{w}}{\partial \nu}\right) d s \\
& =\int_{\partial D}\left(\left[\left.\frac{\partial \bar{v}}{\partial \nu}\right|_{+}-\left.\frac{\partial \bar{v}}{\partial \nu}\right|_{-}\right] \bar{w}-\left[\left.v\right|_{+}-\left.v\right|_{-}\right] \frac{\partial \bar{w}}{\partial \nu}\right) d s \\
& =-\int_{\partial D} \overline{k^{2}\left(n_{2}-1\right)(\mathbf{h} \cdot \nu) u w} d s
\end{aligned}
$$

i.e.,

$$
(M(\phi), h)_{X_{N}}=-\int_{\partial D} \overline{k^{2}\left(n_{2}-1\right)[h(\cos t, \sin t) \cdot \nu] u w} d s .
$$

Hence one has the desired result (3.3).

Consequently, the following theorem holds for the gradient of $F$.

Theorem 3.1. Denote by $w$ the solution of the boundary value problem (3.4). Let

$$
q^{l}=-2 \operatorname{Re}\left(\overline{k^{2}\left(n_{2}-1\right) u w}[(\cos t, \sin t) \cdot \nu], \phi_{l}\right)
$$

where $l=0,1, \ldots, 2 N$. Then

$$
\nabla F(\ldots ; \gamma)=\left(q^{0}, q^{1}, \ldots, q^{2 N}\right)+2 \gamma\left(c_{0}, c_{1}, \ldots, c_{2 N}\right)
$$

Similar results hold for other choices of $\Gamma_{i}$ and $\Gamma_{m}$. 


\section{Numerical Algorithm}

\subsection{Finite element method for the direct problem}

To solve the optimization problem by iterative methods, a well-designed direct solver can improve the efficiency significantly. In this section, we describe a finite element method for the scattering problem using a fixed structured mesh.

Let $N_{x}$ and $N_{y}$ be two positive integers. We divide the intervals $[-s, s]$ and $[0, H]$ uniformly

$$
-s=x_{0}<x_{1}<\cdots<x_{N_{x}}=s, \quad 0=y_{0}<y_{1}<\cdots<y_{N_{y}}=H .
$$

$N_{x}$ and $N_{y}$ are chosen such that $h_{x} \approx h_{y}$, where

$$
h_{x}=\frac{2 s}{N_{x}} \quad \text { and } \quad h_{y}=\frac{H}{N_{y}} .
$$

Let $R_{j l}=\left[x_{j-1}, x_{j}\right] \times\left[y_{l-1}, y_{l}\right], j=1, \ldots, N_{x}, l=1, \ldots, N_{y} . \quad R_{j l}$ is then divided into two triangles $T_{j l}^{1}$ and $T_{j l}^{2}$ by the line segment connecting $\left(x_{j-1}, y_{l-1}\right)$ and $\left(x_{j}, y_{l}\right)$ (see Figure 4.1). Denote $\mathcal{T}_{h}$ the obtained triangular mesh for $\Omega_{0}$. Let

$$
D_{N}=\cup\left\{\left(T_{j l}^{1} \cup T_{j l}^{2}\right): j, l \text { such that }\left(x_{j}^{*}, y_{l}^{*}\right) \in D\right\}
$$

where $\left(x_{j}^{*}, y_{l}^{*}\right)$ is the center of $R_{j l}$. In the finite element computation, we use $D_{N}$ instead of $D$. This is plausible provided the mesh size is small enough.

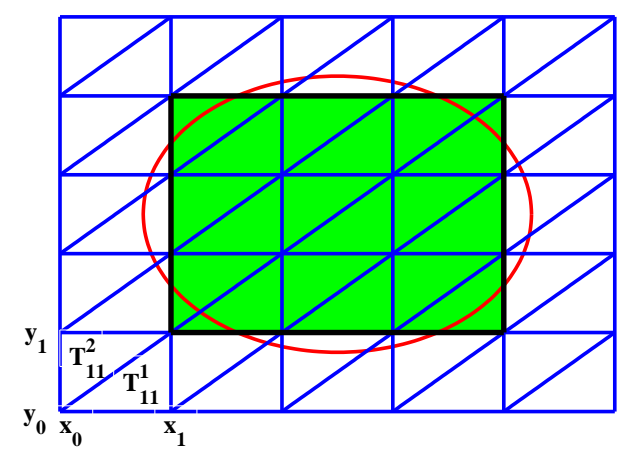

Fig. 4.1. Triangular mesh $\mathcal{T}_{h}$. The shaded region is the approximation $D_{N}$ of $D$.

Let $\left\{\psi_{1}, \psi_{2}, \ldots, \psi_{N}\right\}$ be the linear Lagrange basis for $\mathcal{T}_{h}$ and

$$
u^{s}=\sum_{j=1}^{N} u_{j} \psi_{j} .
$$

Let $\mathbf{u}=\left(u_{1}, u_{2}, \ldots, u_{N}\right)^{T}$ and $A, B, \mathbf{f}$ be given by

$$
\begin{aligned}
& A_{j l}=-\int_{\Omega} \nabla \psi_{l} \cdot \overline{\nabla \psi_{j}}+\int_{\Gamma_{-}} T_{-} \psi_{l} \overline{\psi_{j}} d s+\int_{\Gamma_{+}} T_{+} \psi_{l} \overline{\psi_{j}} d s \\
& B_{j l}=k^{2} \int_{\Omega} n \psi_{l} \overline{\psi_{j}} \\
& \mathbf{f}_{j}=k^{2}\left(1-n_{2}\right) \int_{D_{N}} u^{i} \overline{\psi_{j}}
\end{aligned}
$$


respectively. The discrete problem for the scattered field satisfies

$$
(A+B) \mathbf{u}=\mathbf{f} .
$$

When $D_{N}$ is updated to $D_{N}^{h}, A$ stays the same. Let $T$ be a triangle in $\mathcal{T}_{h}$. From (4.2) and (4.3), we have

$$
\begin{aligned}
& B_{j l}=k^{2} n_{1} \sum_{T \subset \Omega_{0} \backslash D_{N}} \int_{T} \psi_{l} \overline{\psi_{j}}+k^{2} n_{2} \sum_{T \subset D_{N}} \int_{T} \psi_{l} \overline{\psi_{j}}, \\
& \mathbf{f}_{j}=k^{2} \sum_{T \subset D_{N}} \int_{T}\left(1-n_{2}\right) u^{i} \overline{\psi_{j}} .
\end{aligned}
$$

For $D_{N}^{h}$, we have

$$
\begin{aligned}
& B_{j l}^{h}=k^{2} n_{1} \sum_{T \subset \Omega_{0} \backslash D_{N}^{h}} \int_{T} \psi_{l} \overline{\psi_{j}}+k^{2} n_{2} \sum_{T \subset D_{N}^{h}} \int_{T} \psi_{l} \overline{\psi_{j}}, \\
& \mathbf{f}_{j}^{h}=k^{2} \sum_{T \subset D_{N}^{h}} \int_{T}\left(1-n_{2}\right) u^{i} \overline{\psi_{j}} .
\end{aligned}
$$

Defining $K^{+}=D_{N} \backslash D_{N}^{h}$ and $K^{-}=D_{N}^{h} \backslash D_{N}$, we have that

$$
\begin{aligned}
& B_{j l}-B_{j l}^{h}=k^{2}\left(1-n_{2}\right)\left[\sum_{T \in K^{-}} \int_{T} \psi_{l} \overline{\psi_{j}}-\sum_{T \in K^{+}} \int_{T} \psi_{l} \overline{\psi_{j}}\right], \\
& \mathbf{f}_{j}-\mathbf{f}_{j}^{h}=k^{2}\left(1-n_{2}\right)\left[\sum_{T \in K^{-}} \int_{T} u^{i} \overline{\psi_{j}}-\sum_{T \in K^{+}} \int_{T} u^{i} \overline{\psi_{j}}\right] .
\end{aligned}
$$

From $D_{N}$ to $D_{N}^{h}$, one only needs to change a small fraction of the entries of $B$ and $\mathbf{f}$.

\subsection{Initial guess}

The linear sampling method is used to obtain an initial guess. In the following, we briefly sketch the method and refer the readers to [7] for more details.

We first choose a sampling domain $S$ such that $D \subset S$. For the inverse problem in this paper, it is natural to take $S$ to be $\Omega_{0}$. Then we divide the intervals $[-s, s]$ and $[0, H]$ uniformly

$$
\begin{aligned}
& -s=X_{0}<X_{1}<\cdots<X_{M_{x}}=s \\
& 0=Y_{0}<Y_{1}<\cdots<Y_{M_{y}}=H .
\end{aligned}
$$

The sampling points are given by $z_{j l}=\left(X_{j}, Y_{l}\right), j=0,1, \ldots, M_{x}, l=0,1, \ldots, M_{y}$. Let $u^{s}\left(z, z_{0}\right)$ denote the scattered field corresponding to the incident point source $G\left(\cdot, z_{0}\right)$. Let $y_{p}, j=$ $1, \ldots, m$, be $m$ point sources on $\Gamma_{i}$ and $x_{p}, p=1, \ldots, m$, be $m$ measurement locations on $\Gamma_{m}$. At each sampling point $z_{j l}$, we solve the following ill-posed linear integral equation system

$$
\int_{\Gamma_{i}} u^{s}\left(x_{q}, y_{p}\right) g_{j l}\left(y_{p}\right) d y_{p}=G\left(x_{q}, z_{j l}\right), \quad q=1, \ldots, m .
$$

Let $h\left(z_{j l}\right)=\left\|g_{j l}\right\|_{L^{2}\left(\Gamma_{i}\right)}$. 
We choose a contour line of $h$ and use a circle that approximates the contour as the initial guess. Suppose there are $N_{0}$ points on the contour, i.e., $\left(z_{1}^{j}, z_{2}^{j}\right), j=1, \ldots, N_{0}$. The center and radius of the circle are given by

$$
\begin{aligned}
& z_{l}^{0}=\frac{1}{N_{0}} \sum_{j=1}^{N_{0}} z_{l}^{j}, \quad l=1,2 \\
& r_{0}=\frac{1}{N_{0}} \sum_{j=1}^{N_{0}} \sqrt{\left(z_{1}^{j}-z_{1}^{0}\right)^{2}+\left(z_{2}^{j}-z_{2}^{0}\right)^{2}},
\end{aligned}
$$

respectively.

\subsection{Reconstruction algorithm}

Let the incident field be given by

$$
u_{j l}^{i}:=G_{k_{j}}\left(\cdot, z_{l}\right)
$$

with $k_{j}, j=1, \ldots, K$, and $z_{l}, l=1, \ldots, Z$. The reconstruction procedure is summarized as follows.

\section{Reconstruction Algorithm:}

1. Initialization: for the wavenumber $k_{1}$, choose a center $\left(z_{1}^{0}, z_{2}^{0}\right)$ and radius $r_{0}$ using the linear sampling method.

Set $c_{0}=r_{0}, c_{j}=0, j=1,2, \ldots, 2 N$, and set the initial guess $C_{0}=\left(r_{0}, 0, \ldots, 0\right)$.

2. Iteration:

for $j_{0}=1: K$

for $l_{0}=1: Z$

Set $l=1$.

(a) If $l=1$, obtain the numerical approximation of $\nabla F\left(C_{0} ; \gamma\right)$ from Theorem 3.1. If $l \geqslant 2, \nabla F\left(C_{l-1} ; \gamma\right)$ is obtained in the $(l-1)$-th step.

(b) Set a search direction $\xi_{l}=-H_{l-1} \nabla F\left(C_{l-1} ; \gamma\right)$, where $\nabla F\left(C_{l-1} ; \gamma\right)$ is obtained in the $l$-th step, $H_{l-1}=I$ is obtained in the $(l-1)$-th step, $H_{l-1}=I$ if $l=1$.

(c) For $\alpha_{l}^{s}=2^{s}, s \in\{-5,-4, \ldots, 5\}$, define $h_{l}^{s}=\alpha_{l}^{s} \xi_{l}$. Find the largest number $s_{0} \in\{-5,-4, \ldots, 5\}$ and define $h_{l}=h_{l}^{s_{0}}$ such that the Wolfe's condition is satisfied:

$$
F\left(C_{l-1}+h_{l} ; \gamma\right) \leqslant F\left(C_{l-1} ; \gamma\right)+\eta h_{l} \cdot \nabla F\left(C_{l-1} ; \gamma\right)
$$

(d) The new coefficients in the $l$-th step are given by $C_{l}=C_{l-1}+h_{l}$.

(e) Compute $\nabla F\left(C_{l} ; \gamma\right)$ from (3.8). Set $\zeta=\nabla F\left(C_{l} ; \gamma\right)-\nabla F\left(f_{C-1} ; \gamma\right)$ and use the BFGS method to update the new approximate inverse Hessian matrix $H_{l}$. 
(f) Check that if the following four conditions are satisfied: (1) the maximum number of iterations (20 in our algorithm) is reached; $(2) F\left(C_{l} ; \gamma\right)<10^{6}$; (3) $\left|F\left(C_{l-1} ; \gamma\right)-F\left(C_{l} ; \gamma\right)\right|<10^{-8} ;(4)\left\|h_{l}\right\|_{L^{2}[0, L]}<10^{-7}$.

i. Stop if any of the four conditions is satisfied;

ii. Go to (a) if none of the four conditions is satisfied.

Set $C_{0}=C_{l}$.

\section{Numerical Examples}

In this section, we present some numerical examples to show the effectiveness of the proposed method. The point sources are located on $\Gamma_{i}$ and data are measured on $\Gamma_{m}$. We consider the inverse problem when $\Gamma_{i}=\Gamma_{m}=\Gamma_{1} \cup \Gamma_{2}$.

We use multiple frequency data with wavenumbers $1,7,13,19,25,31,37$. The measured data is simulated by the finite element method on a fine unstructured triangular mesh. We add some noise to the computed scattered filed $u^{s}$ to obtain the measured data. Let $\epsilon$ be a normally distributed random number in $[-0.05,0.05]$ and set

$$
u^{\text {meas }}=\left.u^{s}\right|_{\Gamma_{m}}(1+\epsilon) .
$$

For the initial guess by the linear sampling method, we use 8 incident point sources on $\Gamma_{j}, j=1,2$. In the subsequent iterations, we use 2 point sources located on $\Gamma_{1}$ or $\Gamma_{2}$ :

$$
\begin{array}{ll}
\left(0.25,-s_{0}\right),\left(0.75,-s_{0}\right) & \text { on } \Gamma_{1}, \\
\left(0.25, s_{0}\right),\left(0.75, s_{0}\right) & \text { on } \Gamma_{2} .
\end{array}
$$

We set

$$
N_{x}=N_{y}=200, M_{x}=M_{y}=40, N=8 .
$$

The regularization parameter is chosen as $\gamma=10^{-4}$ by trial and error.

\subsection{Near-field data}

Let $s_{0}=0.4$. At $k=1$, we choose a contour at the level

$$
\frac{2}{3} \min _{j, l}\left\{h_{z_{j l}}\right\}+\frac{1}{3} \max _{j, l}\left\{h_{z_{j l}}\right\}
$$

using the linear sampling method. A circle, which approximates the contour, is taken as the initial guess.

Example 5.1. $n_{2}=0.5$. The target $D$ is an ellipse given by

$$
\frac{40}{9} x^{2}+100(y-0.5)^{2}=1 \text {. }
$$

The reconstruction is shown in Figure 5.1.

In Figure 5.1, the left picture shows the initial guess. The dashed line is the initial guess obtained using the linear sampling method. The right picture shows the reconstruction. 

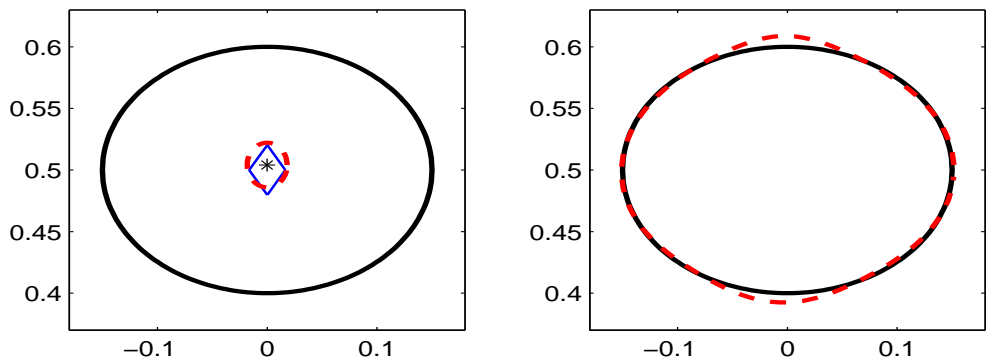

Fig. 5.1. Example 5.1. Left: The solid line is the contour by the linear sampling method. The dashed line is the initial guess. The star is the center of the circle. Right: The dashed line is the reconstructed boundary.

Example 5.2. $n_{2}=2$. The target is a kite with boundary given by

$$
(0.075 \cos t+0.04875 \cos 2 t-0.05,0.1 \sin t+0.5) .
$$

The reconstruction is shown in Figure 5.2.
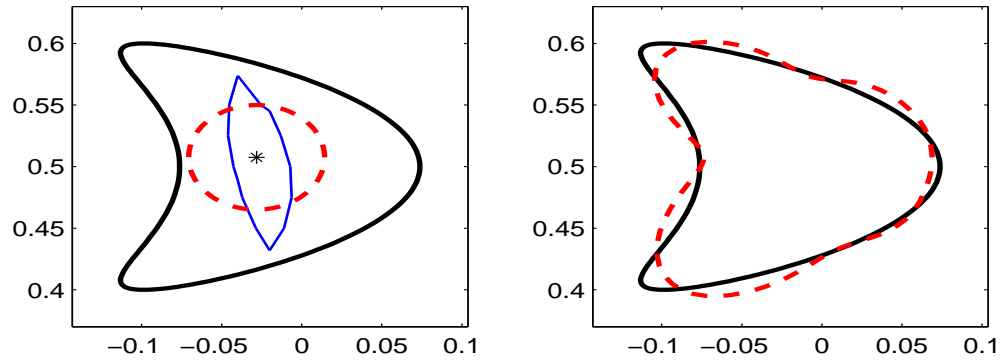

Fig. 5.2. Example 5.2. Left: The solid line is the contour obtained by the linear sampling method. The dashed line is the initial guess. The star is the center of the circle. Right: The dashed line is the reconstructed boundary.

In Example 5.2, the kite is nonconvex and the reconstruction is less satisfactory than convex obstacles (see Figure 5.2). Nonetheless, the numerical method gives a good reconstruction.

Example 5.3. $n_{2}=0.5$. The obstacle is a rectangle with vertices $(-0.05,0.35),(0.15,0.35)$, $(0.15,0.55)$, and $(-0.05,0.55)$. The reconstruction is shown in Figure 5.3.

Example 5.4. $n_{2}=2$. The obstacle is a triangle with vertices $(-0.1,0.35),(0.1,0.35)$, and $(0,0.55)$. The reconstruction is shown in Figure 5.4.

For Example 5.3 and Example 5.4, the boundary of the target is not smooth. The algorithm gives a good reconstruction except the corners, which is expected since we use finite Fourier series to approximate the targets.

The number of the propagating modes plays an important role. If we use larger wavenumbers, more details of the shape could be reconstructed. In Figure 5.5, we show the results using different ending wavenumbers $k_{K}$. 

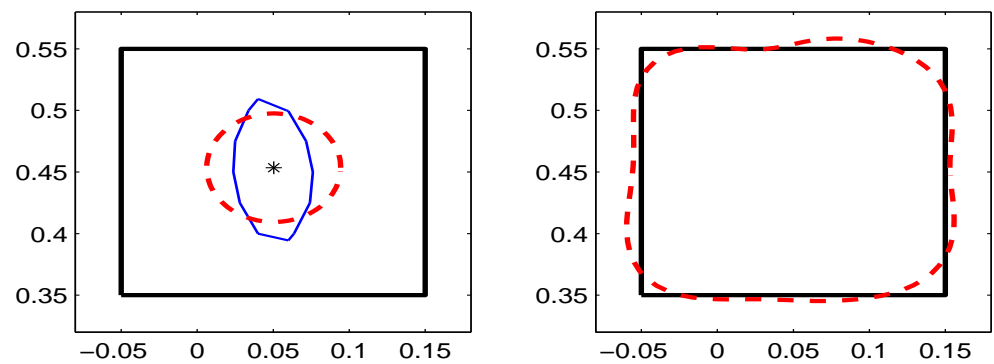

Fig. 5.3. Example 5.3. Left: The solid line is the contour obtained by the linear sampling method. The dashed line is the initial guess. The star is the center of the circle. Right: The dashed line is the reconstructed boundary.
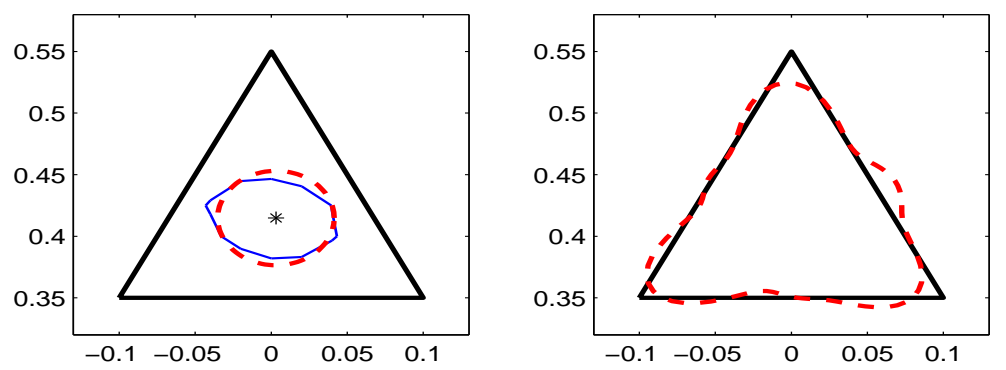

Fig. 5.4. Example 5.4. Left: The solid line is the contour obtained by the linear sampling method. The dashed line is the initial guess. The star is the center of the circle. Right: The dashed line is the reconstructed boundary.

(a)

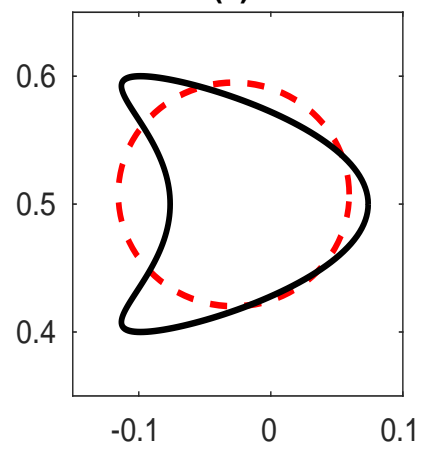

(b)

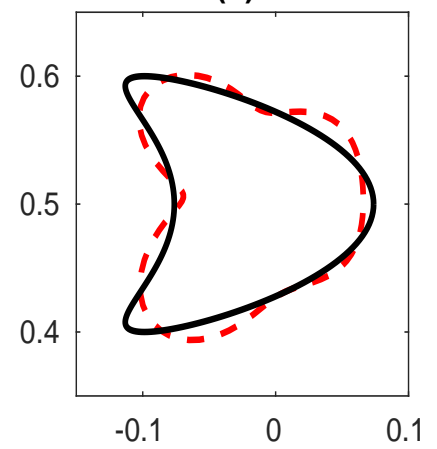

(c)

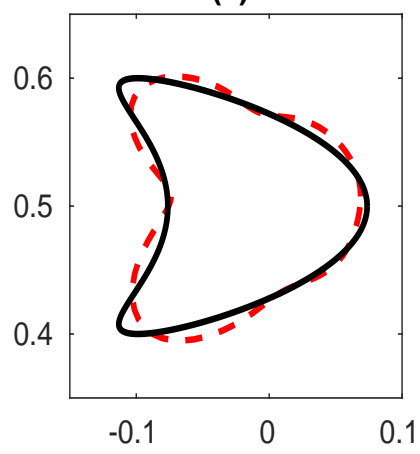

Fig. 5.5. Reconstruction using near-field data with different ending wavenumbers. (a): $k_{K}=13$; (b): $k_{K}=25 ;(\mathrm{c}): k_{K}=37$.

\subsection{Far-field data}

For far-field data, we set $s_{0}=100$. To guarantee propagation modes, $k_{1}=7$. We first compute the scattered data on the line segments $\{ \pm 0.4\} \times[0, H]$. Then the data is extended to $\Gamma_{1}$ and $\Gamma_{2}$ using the analytic extension $\left|x_{1}\right| \rightarrow+\infty$, i.e., there exist coefficients $a_{n}^{(1)}, a_{n}^{(2)} \in \mathbb{C}$ 
such that

$$
\begin{aligned}
& u^{s}\left(x_{1}, x_{2}\right)=\sum_{n \in \mathbb{N}} a_{n}^{(1)} e^{i \beta_{n} x_{1}} \theta_{n}\left(x_{2}\right), \quad \text { when } x_{1}>\max _{t \in[0,2 \pi)}\left\{z_{1}+r(t) \cos t\right\}, \\
& u^{s}\left(x_{1}, x_{2}\right)=\sum_{n \in \mathbb{N}} a_{n}^{(1)} e^{-i \beta_{n} x_{1}} \theta_{n}\left(x_{2}\right), \quad \text { when } x_{1}<\min _{t \in[0,2 \pi)}\left\{z_{1}+r(t) \cos t\right\} .
\end{aligned}
$$

In Figure 5.6, we present the reconstructions using the far-field data for Examples 5.1-5.4.
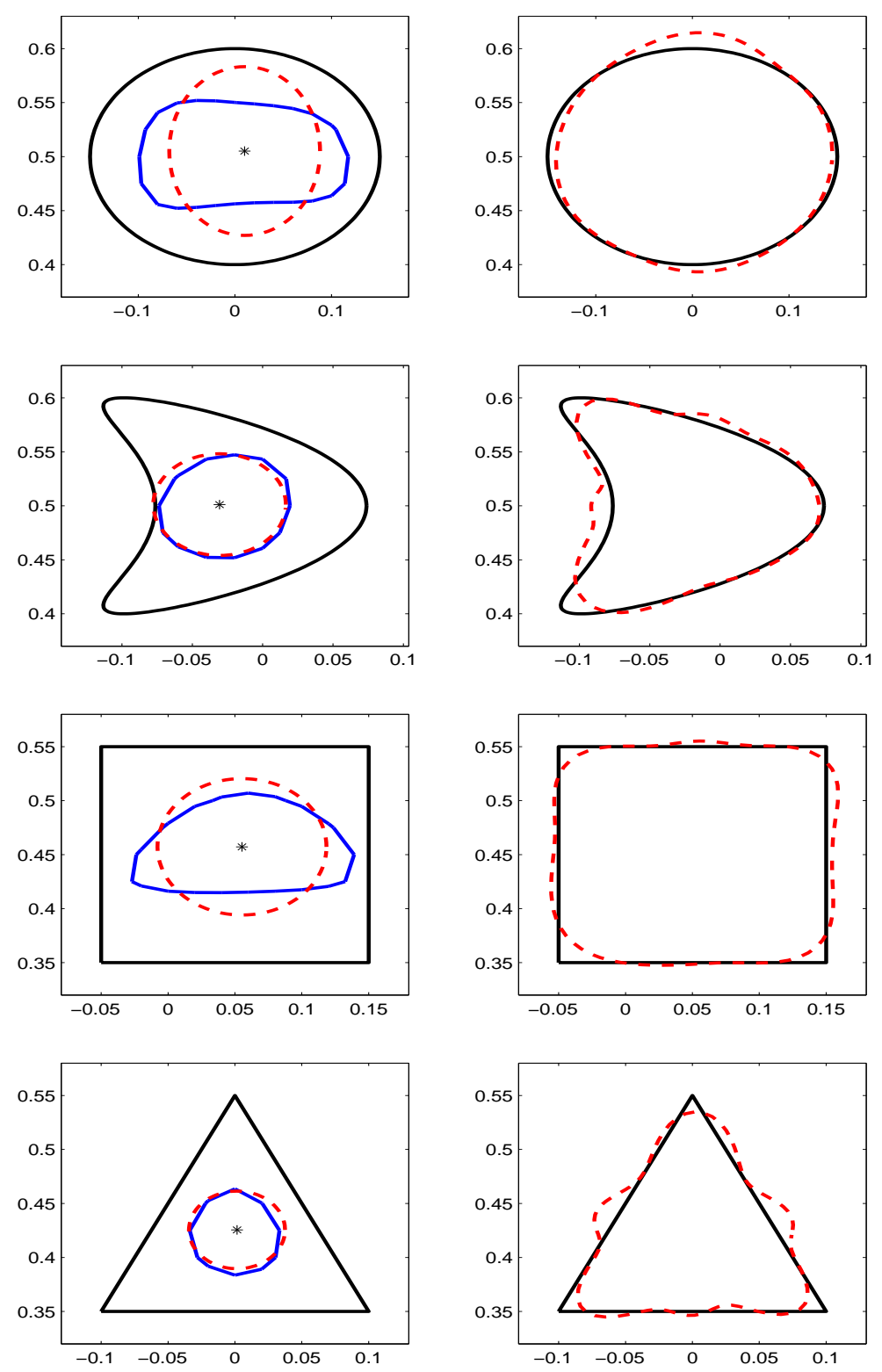

Fig. 5.6. Reconstruction using far-field data. First row: Example 5.1. Second row: Example 5.2. Third row: Example 5.3. Fourth row: Example 5.4. 


\section{Conclusion}

We studied the inverse problem to reconstruct a penetrable obstacle embedded in a planar waveguide from the scattered data. We design a finite element optimization method, which is shown to be effective and efficient. The current paper assumes the index of the refraction of the obstacle is a known constant. We plan to consider the case when the index of refraction is an unknown function.

An advantage of the proposed method is the separation of the boundary given by the Fourier series and the actual boundary used by the finite element method. Given the noise level, an elaborate procedure to decide the size of the fixed mesh would improve the performance of the proposed algorithm significantly, which is under consideration.

Acknowledgments. The work of R. Zhang is supported in part by NSF DMS-1016092/1321391. The work of J. Sun is supported in part by NSF CNIC-1427665 and NSF DMS-1521555.

\section{References}

[1] T. Arens, D. Gintides, and A. Lechleiter, Direct and inverse medium scattering in a 3D homogeneous planar waveguide, SIAM J. Appl. Math., 71 (2011), 753-772.

[2] G. Bao and P. Li, Inverse medium scattering problems in near-field optics, J. Comput. Math., 25 (2007), 252-265.

[3] L. Bourgeois and S. Fliss, On the identification of defects in a periodic waveguide form far field data, Inverse Problems, 30 (2014), 095004.

[4] L. Bourgeois and E. Lunéville, The linear sampling method in a waveguide: a modal formulation, Inverse Problems, 24 (2008), 015018.

[5] L. Bourgeois and E. Lunéville, On the use of sampling methods to identify cracks in acoustic waveguides, Inverse Problems, 28 (2012), 105011.

[6] Z. Chen and G. Huang, Reverse time migration for extended obstacles: acoustic waves, Inverse Problems, 29 (2013), 085005.

[7] D. Colton and A. Kirsch, A simple method for solving inverse scattering problems in the resonance region, Inverse Problems, 12 (1996), 383-393.

[8] S. Dediu and J. R. McLaughlin, Recovering inhomogeneities in a waveguide using eigensystem decomposition, Inverse Problems, 22 (2006), 1227-1246.

[9] M. Ehrhardt, J. Sun and C. Zheng, Evaluation of scattering operators for semi-infinite periodic arrays, Commun. Math. Sci., 7 (2009), Iss. 2, 347-364.

[10] F. Hettlich, Fréchet derivatives in inverse obstacle scattering, Inverse Problems, 11 (1995), 371382.

[11] T. Hohage and C. Schormann, A Newton-type method for a transmission problem in inverse scattering, Inverse Problems, 14 (1998), 1207-1227.

[12] K. Liu, Y. Xu and J. Zou, A parallel radial bisection algorithm for inverse scattering problems, Inverse Probl. Sci. En., 21 (2012), 197-209.

[13] K. Liu and J. Zou, A multilevel sampling algorithm for locating inhomogeneous media, Inverse Problems, 29 (2013), 095003.

[14] K. Liu, Y. Xu and J. Zou, Imaging wave-penetrable objects in a finite depth ocean, Appl. Math. Comput., 235 (2014), 364-376.

[15] K. Liu, Y. Xu and J. Zou, A multilevel sampling method for detecting sources in a stratified ocean waveguide, J. Comput. Appl. Math., 309 (2017), 95-110.

[16] J. Sun, An eigenvalue method using multiple frequency data for inverse scattering problems, Inverse Problems, 28 (2012), 025012. 
[17] J. Sun and C. Zheng, Reconstruction of obstacles embedded in waveguides, Contemp. Math., 586 (2013), 341-350.

[18] Y. Xu, C. Matawa and W. Lin, Generalized dual space indicator method for underwater imaging, Inverse Problems, 16 (2000), 1761-1776.

[19] R. Zhang and J. Sun, An efficient finite element method for grating profile reconstruction. $J$. Comput. Phys., 302 (2015), 405-419. 